\title{
Subconjunctival Dirofilaria repens Infestation: A Light and Scanning Electron Microscopy Study
}

\author{
Henrik A. Melsom ${ }^{1}$, Jørgen A.L. Kurtzhals ${ }^{2}$, Klaus Qvortrup ${ }^{3}$, Ralph Bargum ${ }^{4}$, Toke S. Barfod ${ }^{5}$, \\ Morten la Cour ${ }^{1}$ and Steffen Heegaard ${ }^{*}, 6$
}

\author{
${ }^{I}$ Department of Ophthalmology, Copenhagen University Hospital, Glostrup, Denmark \\ ${ }^{2}$ Centre for Medical Parasitology at Department of Clinical Microbiology, Copenhagen University Hospital \\ (Rigshospitalet) and Department of International Health, Immunology and Microbiology, University of Copenhagen, \\ Denmark \\ ${ }^{3}$ Department of Biomedical Sciences, University of Copenhagen, Denmark \\ ${ }^{4}$ Ralph Bargum's Eye Clinic, Roskilde, Denmark \\ ${ }^{5}$ Department of Infectious Diseases, Copenhagen University Hospital, Rigshospitalet, Copenhagen, Denmark \\ ${ }^{6}$ Eye Pathology Section, Department of Neuroscience and Pharmacology, University of Copenhagen, Denmark
}

\begin{abstract}
Background: To present a case of subconjunctival infestation with Dirofilaria repens which is very rare in Northern Europe.

Methods: A 61-year-old male presented with a swelling and redness of the left supraorbital region migrating to the eyelid and the left eyeball resulting in conjunctival injection, proptosis and diplopia. The patient underwent incisional extraction of a nine cm long worm, which was analysed histologically.

Results: The worm was structureless, greyish-white in colour and measuring nine $\mathrm{cm}$ in length and $0.5 \mathrm{~mm}$ in diameter. Histopathological examination of the worm showed an outer thick, multi-layered cuticle with longitudinal ridges. Beneath the cuticle, a thick muscle layer was observed and internally the intestine and a single reproductive tube containing spermatozoa were noted. Scanning electron microscopy of the worm showed tapered ends, transverse striations and longitudinal ridges at the anterior end. The tail was relatively short with spirally coiled ridges indicating a male Dirofilaria repens.

Conclusions: Humans are an uncommon and accidental host of Dirofilaria repens which is rarely seen in Northern Europe but should be considered as a differential diagnosis to other nematode ocular infections. A travel history is helpful in diagnosing the potential involved organisms. No further treatment is necessary beyond surgical removal since this organism fails to mature and thereby does not cause microfilariaemia in humans.
\end{abstract}

Keywords: Conjunctiva, ocular, microscopy, Dirofilaria repens.

\section{INTRODUCTION}

Human ocular infections with nematodes are rarely seen in Northern Europe since the outdoor temperatures required for normal development are too low though climate changes might eventually allow autochthonous infection in these regions [1].

Of the nematodes, Dirofilaria spp. are relatively common in dogs and various other domestic and wild carnivores. In particular D. repens and D. immitis (heartworm) are increasingly reported as inadvertent human pathogens.

Other species to be considered in subjects clinically suspected of ocular nematode infection include Onchocerca volvulus and Loa Loa. Humans are a natural host of the latter nematodes, meaning they mature completely and result in

*Address correspondence to this author at the Eye Pathology Section, University of Copenhagen, Denmark; Tel: + 45353260 70; Fax: + 4535 3260 80; E-mail: sthe@sund.ku.dk microfilariae, whereas Dirofilaria spp. usually do not mature to adult filaria in the human host. Only one case of D. repens microfilariaemia has been reported [2] suggesting that infiltration in rare cases is possible in the human host.

Filiriasis has long been known to be indigenous of the Mediterranean region, but in recent years an increasing number of cases of autochthonous human dirofilariasis have been reported in Central European countries [3]. In France there are signs of emerging filarial zoonosis North of $46^{\circ}$ latitude, though the majority of confirmed human infections are still occurring in Southern parts of the country [4].

The increasing number of reported human dirofilariasis cases can possibly be explained by an augmented medical awareness and interest amongst investigators along with climate changes and lack of prevention facilitating spread amongst zoonotic reservoirs [5]. During the last decades, non-indigenous nematode infections in general are seen 
more frequently in Northern Europe due to increasing international travel activity.

D. repens reproduces sexually, its lifecycle consisting of five developmental stages requiring a vertebrate main host (usually a dog or cat) and an arthropod intermediate host/vector belonging to a wide range of culicine mosquito species. Sexual maturity and female production of microfilaria occur in the vertebrate host, usually in subcutaneous tissue or in muscle sheaths. The vector through a blood meal then ingests the numerous microfilaria. Following ingestion, microfilariae undergo transformation into third-stage larvae within the intermediate host. The larvae then migrate from the abdomen through the thorax and eventually to the salivary glands of the vector. The transmission of infection to a new vertebrate host may occur at a subsequent blood meal [6]. The immature filaria often migrate subcutaneously; accordingly some patients can describe a migrating location of symptoms giving a clinical clue to the diagnosis. The existing literature suggests that subcutaneous and submucosal, most often subconjunctival, manifestations are the norm of clinical presentation of $D$. repens infection. Pulmonary infection with $D$. repens has been sporadically reported, though this is more typical of human D. immitis infection [7]. The period from exposure to clinical manifestation could be as low as 10 weeks. Here we report on ocular infiltration of a nematode D. repens in a healthy adult male.

\section{MATERIALS AND METHODS}

A 61-year-old male presented with a one-week history of tenderness and irritation starting at the left eyebrow and within days migrating to the eyelid and the left eyeball. The patient had travelled to Brazil and Uruguay the year before presentation and had recently returned from a trip to Italy in the month of September. He had neither known allergies nor prior history of ophthalmological diseases but he had three years earlier been diagnosed with hypertension and hypercholesterolemia for which he received medication.

The patient was treated by his private ophthalmologist for left sided periorbital and conjunctival inflammation with Pivampicillin $700 \mathrm{mg}$ twice daily and six times daily with topical treatment of the affected eye with hydrocortisone, oxytetracycline and polymyxin B. Four days later the patient was referred to the ophthalmological department due to continuous symptoms, demonstrating a slight periorbital oedema, moderate conjunctival injection and a temporal subconjunctival undulating vermiform mass of the left eye (Fig. 1a, b). Decreased elevation and abduction of the left eye along with four mm left sided proptosis was noted.

Closer examination revealed a structure that could be seen moving independently of eye movements confirming the suspicion of a parasitic nematode infection. The ophthalmological examination was otherwise normal as was the systemic examination, particularly no other skin lesions were observed.

With the patient in general anaesthesia, the organism was removed from the left eye through a small conjunctival incision using surgical tweezers. The patient recovered quickly and was given topical antibiotics and steroids prophylactically for the next days until full remission. No adverse effects of the treatment were noted and the postoperative period was uneventful.

\section{RESULTS}

\section{Macroscopic Examination}

The worm appeared with tapered ends, the anterior end being slightly larger in diameter. The worm was otherwise structureless, greyish-white in colour and measuring nine $\mathrm{cm}$ in length and approximately $0.5 \mathrm{~mm}$ in diameter.

\section{Light Microscopy Examination}

The removed worm was fixed in $4 \%$ buffered formaldehyde for more than $24 \mathrm{~h}$ and subsequently embedded in paraffin. Five $\mathrm{mm}$ sections were cut and mounted on glass slides. The sections were stained with haematoxylin-eosin and periodic acid-Schiff. Histopathological examination of the worm showed an outer thick, multilayered cuticle with longitudinal ridges. Beneath the cuticle a thick muscle layer, comprised of tall coelomyarian muscle cells was observed. Internally the intestine and a single reproductive tube containing spermatozoa were seen (Fig. 1f).

\section{Scanning Electron Microscopy (SEM) Examination}

Both ends of the worm were processed for scanning electron microscopy using conventional critical point drying and sputter coating. SEM showed tapered ends, transverse striations and longitudinal ridges at the anterior end, where a mouth was visible at the tip (Fig. 1c). The tail was relatively short with spirally coiled ridges, indicating a male worm, although no spicules could be demonstrated (Fig. 1d). Approximately $50 \mu \mathrm{m}$ from the posterior end the cloaca could be seen (Fig. 1e).

\section{DISCUSSION}

In the case presented a nematode infection quickly became obvious. A few causative species are to be considered after establishing the general diagnosis of a periorbital or ocular nematode in humans: Loa Loa, onchocerciasis (River blindness) along with the dirofilaria species being among the most common. Of the latter $D$. repens is by far the most common in Europe, and in the eye region, subconjunctival and eyelid manifestations are typical of this specific parasite. Our patient could not recollect having been bit by mosquitos, but it can reasonably be assumed that the patient was bitten in an exposed skin region on either an arm or, as the clinical history suggests, the forehead. Orbital or intrabulbar involvement is uncommon in humans. Differential diagnosis can sometimes be difficult to establish through histopathological examination alone, especially when removal in toto is impossible or degeneration of the parasite has set in.

However, it is usually possible to identify the worm to the genus level, and it is not necessary from a clinical point of view to discriminate between Dirofilaria ssp. In order to verify the species identification PCR is the gold standard, and this can be performed on both fresh and formalin-fixed specimens [8].

In cases where identification of the worm is not possible the travel history of the patient along with the anatomical 

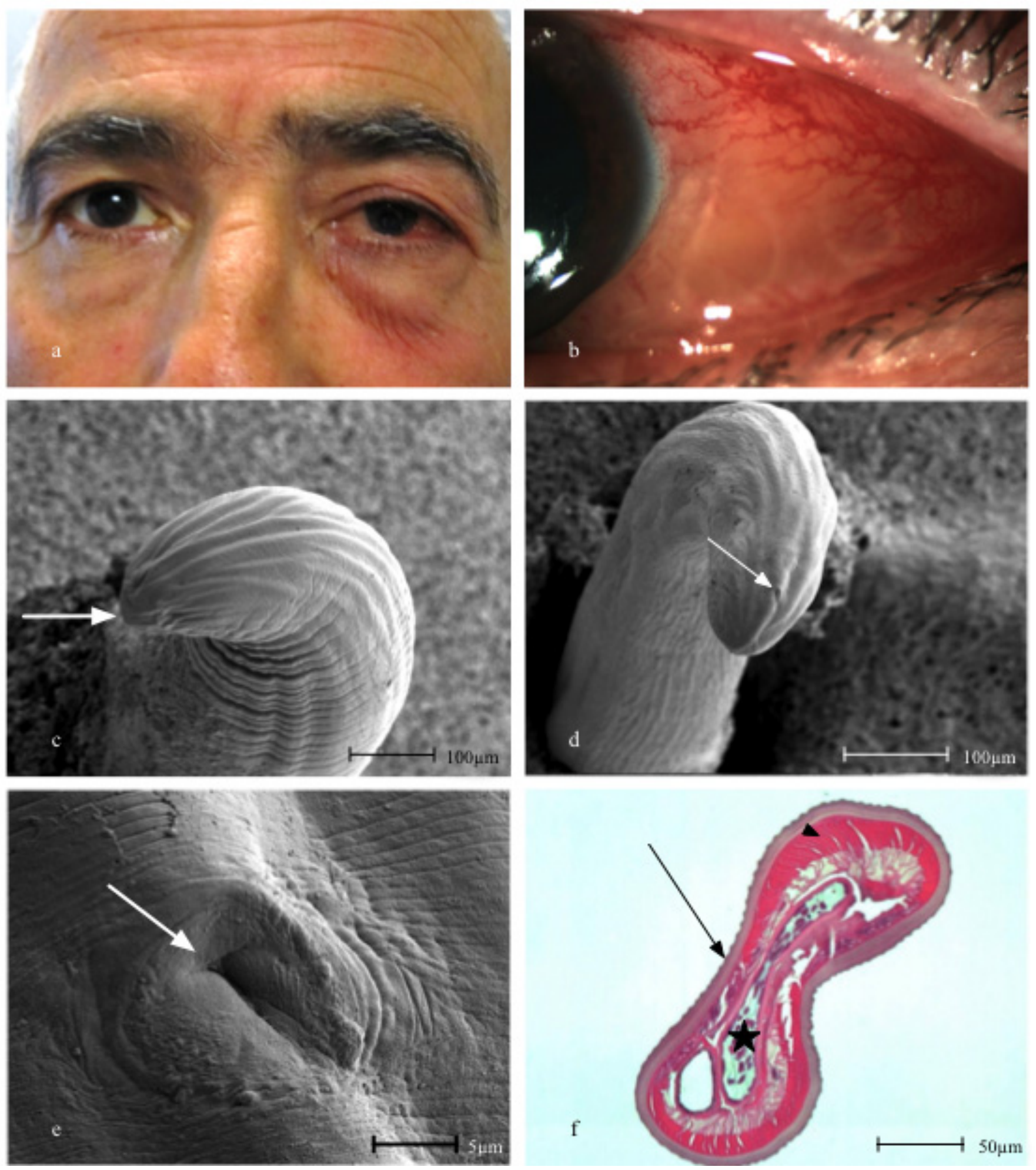

Fig. (1). (a) The 61-year-old patient with slight periorbital swelling and conjunctival injection of the left eye. (b) Close-up showing the worm coiled up in the subconjunctival space of the left eye. (c) Anterior end with mouth (arrow) in scanning electron microscopy (SEM). (d) The tail of the worm showing transverse striations and longitudinal ridges in SEM. Note spiral coiling indicating a male worm and cloaca (arrow). (e) The posterior end of the worm showing the cloaca (arrow) in SEM. (f). Transverse section of the worm with a characteristic multi-layered outer cuticle and external longitudinal ridges (arrow). Beneath the cuticle a thick muscle layer (arrowhead) is observed and internally the intestine and a single reproductive tube (star) (Haematoxylin eosin, X100 original magnification).

location of the invading organism is often helpful. $D$. immitis, for example, is rarely found in the subconjunctival space but more often as a pulmonary or subcutaneous lesion.
Only a few examples of $D$. repens manifestation outside either the subcutaneous or submucosal space have been described [9]. Italy, a country that our patient often and 
recently had visited, is recognized as the region with the highest incidence of human $D$. repens infection in the world [10].

This case demonstrates that ocular manifestations of $D$. repens, although rare in Northern Europe, can be found and should be considered as a differential diagnosis to other nematode ocular infections. A complete travel history is helpful in diagnosing the potential involved organisms. No further treatment beyond surgical removal is necessary since this organism fails to mature and thereby does not cause microfilariaemia in humans.

\section{SUPPORTIVE/SUPPLEMANTARY MATERIAL}

Surgical removal video and it can be viewed on line at publisher's website.

\section{REFERENCES}

[1] Genchi C, Rinaldi L, Mortarino M, Genchi M, Cringoli G. Climate and dirofilaria infection in Europe. Vet Parasitol 2009; 163: 28692.

[2] Nozais JP, Bain O, Gentilini M. A case of subcutaneous Dirofilaria (Nochtiella) repens with microfilaremia originating in Corsica. Bull Soc Pathol Exot 1994; 87: 183-5.
[3] Szénási Z, Kovács AH, Pampiglione S, et al. Human dirofilariosis in Hungary: An emerging zoonosis in Central Europe. Wien Klin Wochenschr 2008; 120: 96-102.

[4] Raccurt CP. Human dirofilariasis in France: new data confirming the human transmission of Dirofilaria repens to the north of the 46 degree north latitude. Med Trop (Mars) 2000; 60: 308-9.

[5] Genchi C, Rinaldi L, Cascone C, Mortarino M, Cringoli G. Is heartworm disease really spreading in Europe? Vet Parasitol 2005; 133: $137-48$.

[6] Cancrini G, Pietrobelli M, Frangipane di Regalbono AF, Tampieri MP, della Torre A. Development of Dirofilaria and Setaria nematodes in Aedes albopictus. Parassitologia 1995; 37: 141-5.

[7] Abdel-Rahman SM, Mahmoud AE, Galal LA, Gustinelli A, Pampiglione S. Three new cases of human infection with Dirofilaria repens, one pulmonary and two subcutaneous, in the Egyptian governorate of Assiut. Ann Trop Med Parasitol 2008; 102: 499-507.

[8] Rivasi F, Boldorini R, Criante P, Leutner M, Pampiglione S. Detection of Dirofilaria (Nochtiella) repens DNA by polymerase chain reaction in embedded paraffin tissues from two human pulmonary locations. APMIS 2006; 114: 567-74.

[9] Pampiglione S, Rivasi F. Human dirofilariasis due to Dirofilaria (Nochtiella) repens: An update of world literature from 1995 to 2000. Parassitologia 2000; 42: 231-54.

[10] Pampiglione S, Rivasi F, Angeli G, et al. Dirofilariasis due to Dirofilaria repens in Italy, an emergent zoonosis: report of 60 new cases. Histopathology $2001 ; 38: 344-54$ 